Research Article

\title{
The Effect of Liraglutide on Epicardial Adipose Tissue in Type 2 Diabetes
}

\author{
Na Zhao $\mathbb{D}$, Xiaoying Wang, Yongbo Wang, Junjie Yao, Chunhong Shi, Jianling Du, \\ and Ran Bai
} Department of Endocrinology and Metabolism, The First Affiliated Hospital of Dalian Medical University, Dalian,
Liaoning Province, China

Correspondence should be addressed to Ran Bai; bairan5556@163.com

Received 21 January 2021; Revised 18 October 2021; Accepted 23 October 2021; Published 16 November 2021

Academic Editor: Raffaele Marfella

Copyright (c) $2021 \mathrm{Na}$ Zhao et al. This is an open access article distributed under the Creative Commons Attribution License, which permits unrestricted use, distribution, and reproduction in any medium, provided the original work is properly cited.

\begin{abstract}
Objective. To study the effect of liraglutide on the thickness of epicardial adipose tissue (EAT) in type 2 diabetes mellitus (T2DM) patients with abdominal obesity. Methods. Abdominal obesity T2DM patients with poor glycemic control were collected and treated with liraglutide. The changes of blood glucose, blood lipid, waist circumference, body mass index (BMI), and EAT thickness were compared after 3 months of treatment with liraglutide. Cardiac magnetic resonance imaging (MRI) was used to measure EAT thickness. Results. After 3 months of treatment with liraglutide, glycosylated hemoglobin $\left(\mathrm{HbA}_{1 \mathrm{c}}\right)$ decreased from $9.81 \pm 1.46 \%$ to $6.94 \pm 1.29 \%(95 \% \mathrm{CI}=2.14-3.59, p<0.001)$. The weight decreased from $91.67 \pm 16.29 \mathrm{~kg}$ to $87.29 \pm 16.43 \mathrm{~kg}$ $(95 \% \mathrm{CI}=2.97-5.79, p<0.001)$. Waist circumference before treatment was $103.69 \pm 9.14 \mathrm{~cm}$, and after treatment was 96.42 $\pm 8.42 \mathrm{~cm}(95 \% \mathrm{CI}=5.04-9.50, p<0.001)$. Total cholesterol $(\mathrm{TC})$, triglyceride $(\mathrm{TG})$, and low-density lipoprotein cholesterol (LDL-C) were significantly lower than those before treatment. TC decreased from $5.34 \pm 1.05 \mathrm{mmol} / \mathrm{L}$ to $4.86 \pm 0.97 \mathrm{mmol} / \mathrm{L}$ $(95 \% \mathrm{CI}=0.15-0.82, p<0.001)$. TG was $1.89(1.48-3.17)$ and then to $1.92 \pm 0.69(p=0.03)$. LDL-C decreased from $3.39 \pm$ $0.84 \mathrm{mmol} / \mathrm{L}$ to $3.01 \pm 0.74 \mathrm{mmol} / \mathrm{L}(95 \% \mathrm{CI}=0.17-0.59, p=0.001)$. HDL-C increased by $1.7 \%$ after treatment, with no significant difference $(p=0.062)$. More importantly, the thickness of EAT decreased from 5.0 (5.0-7.0) $\mathrm{mm}$ to $3.95 \pm 1.43$ $\mathrm{mm}(p<0.001)$ after liraglutide administered for 3 months. Conclusion. Liraglutide significantly reduces EAT thickness in T2DM with abdominal obesity, which provides theoretical support for the cardiovascular benefits of liraglutide.
\end{abstract}

\section{Introduction}

Type 2 diabetes mellitus (T2DM) is a metabolic syndrome with disorders of glucose, protein, and fat caused by deficiency of insulin secretion and/or insulin function. It is often accompanied by obesity. Obesity, especially visceral fat accumulation, can lead to islet dysfunction, insulin resistance (IR), prediabetes, T2DM, and inflammatory reaction and increase the incidence of coronary heart disease, hypertension, and other events $[1,2]$. Quantification and reduction of visceral fat represent effective methods to identify high-risk individuals and reduce their cardio-metabolic risk of T2DM patients [3]. Epicardial adipose tissue (EAT), as visceral fat, has attracted much attention due to its close relationship with myocardium and coronary artery [4]. The increase of EAT may represent a chronic inflammatory injury and is related to coronary artery disease [5], and T2DM patients had significantly thicker EAT [6]. Liraglutide is a glucagon-like peptide1 (GLP-1) analog which can activate GLP-1 receptor (GLP1R). In T2DM patients, studies suggested that GLP-1 receptor agonist (GLP-1 RA) could redistribute adipose tissue deposits and reduce visceral fat $[7,8]$. Additionally, liraglutide can reduce the risk of cardiovascular events. Our present study was to explore whether, in addition to its effects on blood glucose and blood lipids, liraglutide reduces cardiovascular events by acting on EAT in T2DM with abdominal obesity. 


\section{Design and Methods}

2.1. Participant Identification. T2DM patients with abdominal obesity who had poor blood glucose control in Endocrinology Department of Jinpu District, the First Affiliated Hospital of Dalian Medical University from November 2016 to February 2018 were consecutive collected. Participants' inclusion criteria were as follows: (1) aged between 18 and 70 years, (2) body mass index $(\mathrm{BMI})>25 \mathrm{~kg} / \mathrm{m}^{2}$ and waist circumference $\geq 90$ $\mathrm{cm}$ in males and $\geq 85 \mathrm{~cm}$ in females, (3) glycosylated hemoglobin $\left(\mathrm{HbA}_{1 \mathrm{c}}\right)$ was between 7 and $11 \%$, and (4) the basic drugs were metformin and/or $\alpha$-glucosidase inhibitors and/or insulin at the time of enrollment and met the liraglutide indications. Exclusion criteria are as follows: (1) inflammatory bowel disease and diabetic gastroparesis; (2) pregnant or breastfeeding women; (3) moderate to severe liver dysfunction (Child's-Pugh score); (4) acute or chronic renal insufficiency, glomerular filtration rate $(\mathrm{GFR})<60 \mathrm{ml} /\left(\min \times 1.73 \mathrm{~m}^{2}\right)$; (5) history of thyroid tumor or pancreatitis; (6) diabetes with acute complications or with tumor; (7) electronic implants such as cardiac pacemaker, insulin pump, and magnetic metal; and (8) uncontrollable involuntary movement, claustrophobia patients, and so on who are not suitable for MRI examination. With the approval of the medical ethics committee, all participants were informed of the possible side effects of liraglutide and provided informed consent.

2.2. Drugs and Dosage. Liraglutide (Victoza, manufactured by Novo Nordisk, Denmark) was injected subcutaneously once a day for 3 months, starting from $0.6 \mathrm{mg} /$ day and increasing to $1.2 \mathrm{mg} /$ day in 3-5 days if there was no adverse. The curative effect of liraglutide was evaluated by monitoring fasting plasma glucose (FPG) and 2-hour blood glucose after three meals every day and before bedtime and early morning if necessary. For patients with poor hypoglycemic effect, the dose was further increased to $1.8 \mathrm{mg} /$ day.

2.3. Biochemical Blood Tests. The patients kept a light diet for three days and fasting water for 12 hours before the blood tests. Blood samples were drawn in the next morning. FPG, $\mathrm{HbA}_{1 \mathrm{c}}$, total cholesterol (TC), triglyceride (TG), lowdensity lipoprotein cholesterol (LDL-C), and high-density lipoprotein cholesterol (HDL-C) were tested ahead of and after 3 months liraglutide being administered by automatic biochemical analyzer (Hitachi 7600, Japan). The 2-hour postprandial blood glucose (2hPBG) was measured by Accu-Chek advantage electronic sensing portable blood glucose meter (Roche, Germany).

2.4. EAT Thickness Measurement. EAT thickness was measured before and after 3-month liraglutide treatment by $1.5 \mathrm{~T}$ cardiac MRI (platform HDxt; General Electric Medical Systems, Waukesha, WI, USA). Scanning parameters were TR $3.6 \mathrm{~ms}$, TE1.6ms, turning angle $50^{\circ}$, bandwidth $125 \mathrm{kHz}$, FOV $350 \mathrm{~mm} * 350 \mathrm{~mm}$, matrix $192 \times 224$, layer thickness $10 \mathrm{~mm}$, and layer spacing 0 . EAT thickness was measured at the right ventricle late diastolic phase with four-chamber echocardiography by two well-experienced radiologists. All cardiac MRI scans were performed by the same radiographer.
2.5. Statistical Analysis. The SPSS 19.0 software was used for statistical analysis. Normal distributed data were presented as the mean \pm standard deviation $(M \pm \mathrm{SD})$. Abnormally distributed data were presented as the median (interquartile range). Paired $t$-test and Wilcoxon test were used to compare the differences of data before and after liraglutide treatment. It is considered statistically significant when the $p$ value was less than 0.05 .

\section{Results}

3.1. Subject Enrollment. The basic demographic characteristics of 21 out of 27 subjects who completed the trial are shown in Table 1. After the injection of liraglutide, the patients had varying but tolerable degrees of nausea and loss of appetite. For most of the patients, the above symptoms gradually disappeared without drug intervention after 1-3 days. No severe hypoglycemia was observed during the whole trial, while among the 27 subjects, 5 were withdrawn from the group because of intolerable gastrointestinal reaction, and 1 was withdrawn because of skin allergy at the injection site. The average dose of liraglutide in 3 months was $1.2 \mathrm{mg} /$ day.

3.2. FPG, $2 h P B G$, and $H b A_{1 c}$ Decreased. After the patients received 3 months of liraglutide, compared with that before liraglutide, FPG decreased from $10.31 \pm 2.41 \mathrm{mmol} / \mathrm{L}$ to 7.17 $\pm 1.28 \mathrm{mmol} / \mathrm{L}(95 \% \mathrm{CI}=2.07-4.20, p<0.001)$, and $2 \mathrm{hPBG}$ decreased from $12.68 \pm 3.33 \mathrm{mmol} / \mathrm{L}$ to $9.12 \pm 1.37 \mathrm{mmol} / \mathrm{L}$ $(95 \% \mathrm{CI}=2.05-5.07, p<0.001)$, shown in Figure 1(a). What is more, $\mathrm{HbA}_{1 \mathrm{c}}$ decreased from $9.81 \pm 1.46 \%$ to $6.94 \pm 1.29 \%$ $(95 \% \mathrm{CI}=2.14-3.59, p<0.001)$, shown in Figure 1(b). FPG, $2 \mathrm{hPBG}$, and $\mathrm{HbA}_{1 \mathrm{c}}$ were dropped by $30.5 \%, 28.1 \%$, and $29.3 \%$ on average.

3.3. Weight, Waist Circumference, and BMI Decreased. The patients' average body weight was $91.67 \pm 16.29 \mathrm{~kg}$ before liraglutide treatment and $87.29 \pm 16.43 \mathrm{~kg}$ after treatment, dropped by $4.38 \mathrm{~kg}$ on average $(95 \% \mathrm{CI}=2.97-5.79, p<$ 0.001 ). BMI decreased on average by $1.49 \mathrm{~kg} / \mathrm{m}^{2}$ from $30.97 \pm 4.04 \mathrm{~kg} / \mathrm{m}^{2}$, and after treatment was $29.48 \pm 4.08$ $\mathrm{kg} / \mathrm{m}^{2}(95 \% \mathrm{CI}=1.02-1.98, p<0.001)$. Waist circumference decreased by $7.27 \mathrm{~cm}$ on average from $103.69 \pm 9.14$ $\mathrm{cm}$ to $96.42 \pm 8.42 \mathrm{~cm} \quad(95 \% \mathrm{CI}=5.04-9.50, p<0.001)$. Results are shown in Figure 2.

3.4. Effect of Liraglutide on Blood Lipid Level. TC decreased from $5.34 \pm 1.05 \mathrm{mmol} / \mathrm{L}$ to $4.86 \pm 0.97 \mathrm{mmol} / \mathrm{L}$ $(95 \% \mathrm{CI}=0.15-0.82, p<0.001), \mathrm{TG}$ decreased from 1.89 (1.48-3.17) and then to $1.92 \pm 0.69(p=0.03)$, and LDL-C decreased from $3.39 \pm 0.84 \mathrm{mmol} / \mathrm{L}$ to $3.01 \pm 0.74 \mathrm{mmol} / \mathrm{L}$ $(95 \% \mathrm{CI}=0.17-0.59, p=0.001)$ (Figure 3). HDL-C increased by $1.7 \%$ on average from $0.96(0.89-1.45) \mathrm{mmol} / \mathrm{L}$ to $1.19 \pm$ $0.25 \mathrm{mmol} / \mathrm{L}$, but the difference was not statistically significant $(p=0.062)$.

3.5. EAT Thickness Decreased. The EAT thickness was 5.0 (5.0-7.0) $\mathrm{mm}$ to $3.95 \pm 1.43 \mathrm{~mm}$ after treatment measured by MRI, with an average decrease of $1.62 \mathrm{~mm}$. The changes suggested that liraglutide can significantly reduce EAT thickness $(p<0.001$, Figure 4$)$. 
TABLE 1: Demographic characteristics of T2DM patients.

\begin{tabular}{lc}
\hline Characteristics & T2DM patients \\
\hline Number & 21 \\
Gender (female/male) & $6 / 15$ \\
Age (mean $\pm \mathrm{SD}$, years) & $42.86 \pm 11.15$ \\
Height (mean $\pm \mathrm{SD}, \mathrm{cm})$ & $171.62 \pm 8.49$ \\
Weight $($ mean $\pm \mathrm{SD}, \mathrm{kg})$ & $91.67 \pm 16.30$ \\
$\mathrm{BMI}\left(\right.$ mean $\left.\pm \mathrm{SD}, \mathrm{kg} / \mathrm{m}^{2}\right)$ & $30.97 \pm 4.04$ \\
\hline
\end{tabular}

\section{Discussion}

Liraglutide, as a GLP-1 analog, has 97\% homology structurally with endogenous human GLP-1 [9]. GLP-1 receptor (GLP1R) mainly exists in fat, liver, gastrointestinal tract, kidney, cardiovascular, and central nervous system [10]. Liraglutide activates GLP-1R to promote insulin secretion of pancreatic $\beta$ cells, reduce body weight, promote vasodilation, and reduce the level of atherosclerotic risk factors in endothelial cells [11-13]. Our results showed that FPG, 2hPBG, and $\mathrm{HbA}_{1 \mathrm{c}}$ of T2DM patients with abdominal obesity were significantly decreased after liraglutide treatment for 3 months, and the body weight, waist circumference, BMI, TG, TC, and LDL-C levels were also significantly decreased. Similar to the results of the present study, liraglutide can effectively reduce $\mathrm{HbA}_{1 \mathrm{c}}$ [14] and body weight [9]. Aoki and his colleagues found that the non-HDL-C and calculated TC were decreased significantly after administrating of liraglutide for 3 months [15]. In addition, in T2DM patients receiving standard care, liraglutide can reduce the risk of cardiovascular events compared with the placebo group [16], but the specific reason is yet not fully clear.

Epicardial adipose tissue (EAT), characterized by brown adipose tissue, is closely connected with the myocardium and coronary artery without fascia barrier. Under physiological conditions, EAT can mitigate the impact, absorb fatty acids, and protect myocardium and coronary arteries from cold injury [17]. It can balance the secretion of proinflammatory and anti-inflammatory factors, regulate the cardiovascular system, and play an important role in obesity-related inflammation and atherosclerosis $[18,19]$. Scholars have also confirmed that EAT is positively correlated with obesity, impaired fasting blood glucose regulation, insulin resistance, metabolic syndrome, hypertension, and coronary heart disease through adipocyte hypertrophy and hyperplasia [20, 21]. T2DM patients had significantly thicker EAT associated with cardiac systolic dysfunction [6]. What is more, EAT volume in patients with coronary heart disease with abdominal obesity is significantly higher than that in patients without coronary heart disease [22]. Therefore, for T2DM patients with abdominal obesity, we should not only effectively control blood glucose levels but also monitor EAT levels.

The monitoring of EAT included abdominal circumference, echocardiography, multislice computed tomography (MCT), and magnetic resonance imaging (MRI). Among them, abdominal circumference is the cheapest and most easily obtained visceral fat marker, but it lacks sensitivity and specificity. Echocardiography is noninvasive, reliable, easy to repeat, and relatively accurate, but it cannot obtain a linear measurement value in a single position. Because of its high accuracy, low variability, and high repeatability, MRI results can be used as the reference standard for quantification of EAT and it is considered as the gold standard for measuring visceral fat [23-25]. There are few studies on the measurement of EAT by cardiac MRI. By using cardiac MRI measurements, we found that after 3 months of liraglutide treatment, the thickness of EAT decreased significantly along with the decrease of waist circumference. Similar to the results of this study, Bouchi et al. found that liraglutide can reduce visceral fat and improve the quality of life of diabetic patients [8]. Chinese scholars, in studies of T2DM patients with obesity on the basis of metformin treatment combined with liraglutide, found that it can improve islet beta cell function and significantly reduce visceral fat levels [26]. In addition, some scholars found that receiving metformin for at least 6 months could alleviate the inflammatory burden of coronary perivascular adipose tissue in prediabetic patients and reduce the level of inflammation in coronary artery fat in patients with prediabetes with acute myocardial infarction (AMI) who underwent acute coronary artery bypass grafting (CABG) [27]. Metformin also influences cardiomyocyte lipid. Scholars found that the lipid accumulation and lipotoxic factors of cardiac myocytes were decreased when receiving metformin in hearts transplanted study [28]. Metformin also was used in the basic medication of the present study. Does metformin synergize or enhance the regulation of liraglutide on EAT in this study? However, Iacobellis and colleagues found that there was no EAT reduction in the metformin monotherapy group in subjects with type 2 diabetes at 3 and 6 months study, whereas EAT decreased significantly when treated with additional liraglutide measured by ultrasound [29]. What is more, EAT decreased significantly by GLP-1RA like semaglutide or dulaglutide in a dose-dependent way, and there was no EAT thickness reduction in the metformin group in a 12-week parallel group study [3], and that Jonker and colleagues also found that metformin treatment did not affect the change of pericardial fat (including epicardial fat and paracardial fat) volume [30]. Nevertheless, Ziyrek et al. found that metformin monotherapy for 3 months could reduce the thickness of EAT by echocardiography [31]. What is more, Bizino et al. found that liraglutide mainly reduced subcutaneous fat but not visceral or epicardial fat compared with control groups at a secondary endpoint study [32]. The results were not consistent with the conclusion of the present study. Due to the differences of sample size, race, measuring instrument, follow-up time, and the basic drugs, the results obtained by scholars are inconsistent. We suspect that both liraglutide and metformin are involved in the regulation of visceral fat. Metformin may act on molecular level through the active inflammatory response gradually in visceral fat, while liraglutide can regulate visceral fat more obviously, which was shown in the decrease of EAT thickness in our study. GLP-1R in EAT is related to fatty acid oxidation and white fat browning [33], and there may be a brown fat whitening transition in adipocyte hypertrophy and hyperplasia in EAT of abdominal obesity T2DM patients. The mechanism of liraglutide in reducing EAT 


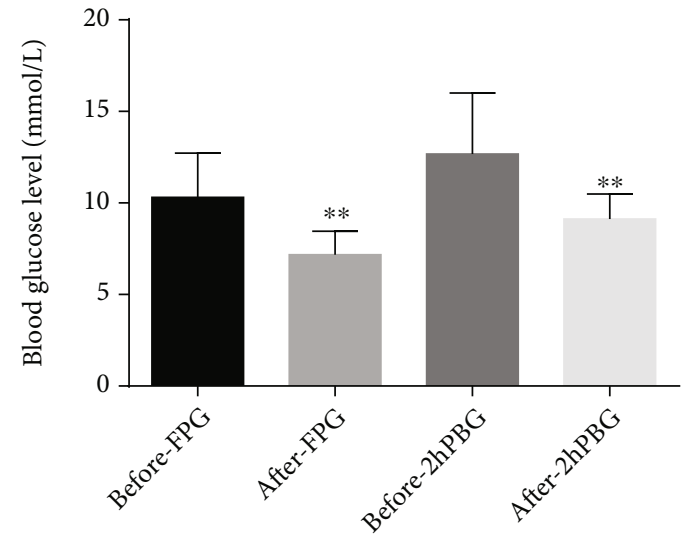

(a)

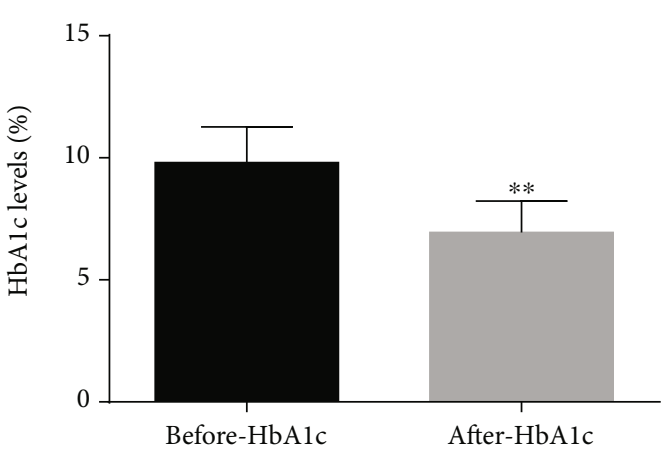

(b)

Figure 1: After liraglutide was added, FPG, 2hPBG, and $\mathrm{HbA}_{1 \mathrm{c}}$ decreased significantly. (a, b) FPG and HbA $\mathrm{Hc}_{\mathrm{c}}$ were tested by automatic biochemical analyzer, and $2 \mathrm{hPBG}$ was measured by Accu-Chek advantage electronic sensing portable blood glucose meter $\left({ }^{* *} p<0.01\right)$.

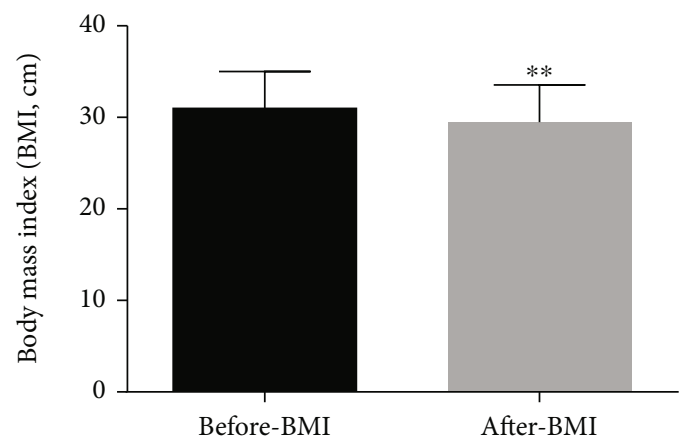

(a)

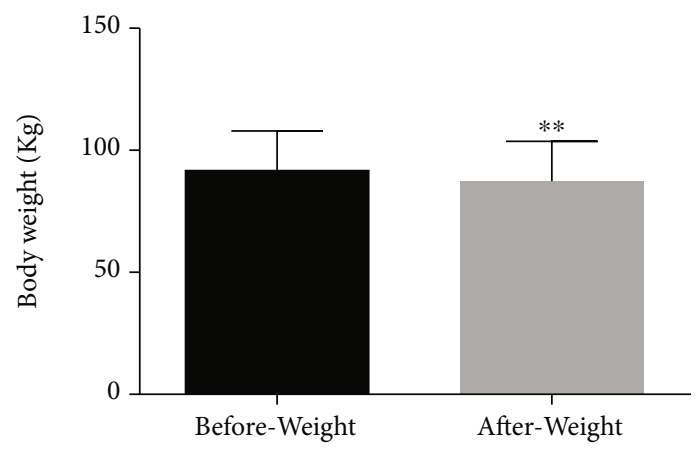

(b)

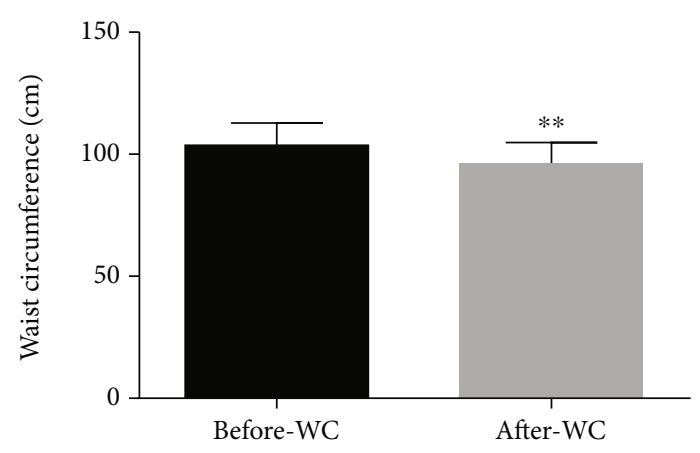

(c)

FIGURE 2: After liraglutide treatment, the weight, waist circumference (WC), and BMI decreased $\left({ }^{* *} p<0.01\right)$.

considered to be that it reverses the whitening of brown fat in EAT by stimulating GLP-1R after administration of liraglutide, thus reducing the thickness of EAT and protecting cardiovascular. Diabetes patients showed obvious early and progressive myocardial lipid accumulation. Whether or not liraglutide and metformin have the same alleviating effect on diabetic cardiomyopathy need further investigation.

Scholars also found that liraglutide can alleviate endotoxemia-related microvascular thrombosis through GLP1 receptor signal-mediated immune regulation without affecting blood glucose or $\mathrm{HbA}_{1 \mathrm{c}}$ level, to prevent systemic inflamma- tion, vascular dysfunction, and end organ injury, which has potential clinical significance for the treatment of sepsis [34]. GLP-1R activation reduces vascular inflammation by selectively acting on endothelial cells rather than myeloid cells to reduce the cardiovascular complications of arterial hypertension [35]. Liraglutide could regulate sepsis-induced endothelial dysfunction by reducing vascular inflammation and oxidative stress [36]. Moreover, in the early stage of thrombosis, a specific antithrombotic therapy may inhibit the specific target pathway of coagulation cascade and change the thrombosis process to improve the clinical outcome of patients [37], while it is still 


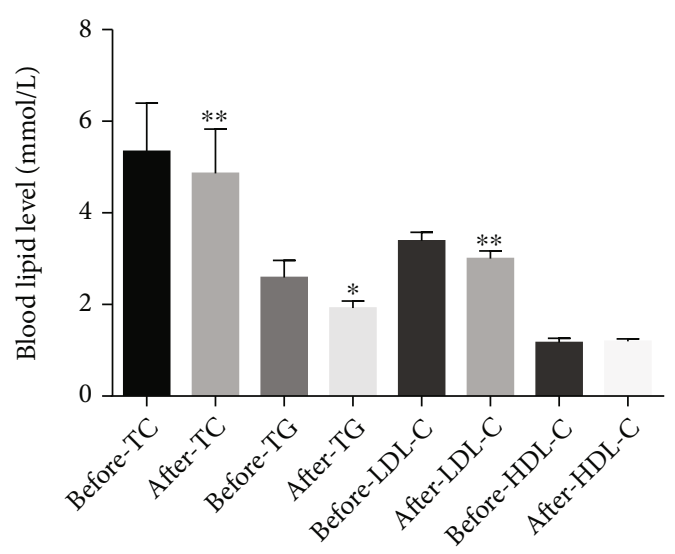

Figure 3: Changes of blood lipids after liraglutide treatment. The levels of total cholesterol (TC), triglyceride (TG), low-density lipoprotein cholesterol (LDL-C), and high-density lipoprotein cholesterol (HDL-C) were measured by automatic biochemical analyzer $\left({ }^{*} p<0.05\right.$ and $\left.{ }^{* *} p<0.01\right)$.

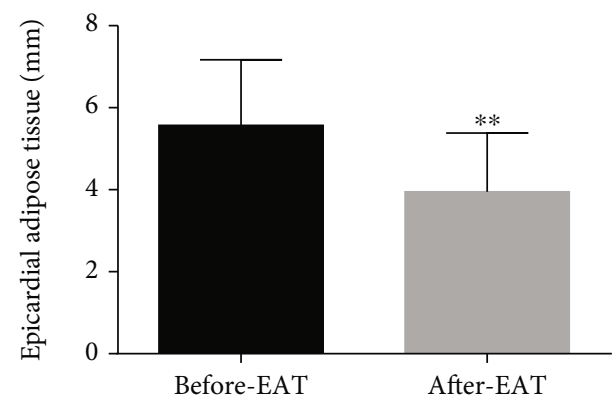

FIGURE 4: EAT thickness was decreased when liraglutide was introduced. $1.5 \mathrm{~T}$ cardiac magnetic resonance imaging (MRI) was used to measure the thickness of EAT $\left({ }^{* *} p<0.01\right)$.

unclear and need to be further studied whether and how liraglutide could be used to improve the therapy.

To sum up, this study explored the decrease of FPG, 2hPBG, $\mathrm{HbA}_{1 \mathrm{c}}$, body weight, waist circumference, BMI, TG, TC, and LDL-C levels in abdominal obesity T2DM patients after treatment with liraglutide. Cardiac MRI was used to measure the thickness of EAT. It was found that the epicardial adipose tissue thickness was significantly reduced after 3 months of liraglutide treatment, in addition to effective hypoglycemic and lipid-lowering effects, providing theoretical support that liraglutide could effectively contribute to the protection of the coronary arteries in T2DM patients with abdominal obesity.

\section{Data Availability}

All data included in this study are available upon request by contact with the corresponding author.

\section{Conflicts of Interest}

The authors declare that they have no conflicts of interest.

\section{Acknowledgments}

We would like to appreciate the support from professor Zhiyong Li and his colleagues in MRI data measurement.

\section{References}

[1] G. Yan, "Protective effects of liraglutide on cardiovascular system," Chinese Journal of Endocrinology and Metabolism, vol. 29, no. 10, pp. 917-920, 2013.

[2] F. Santilli, P. G. Simeone, M. T. Guagnano et al., "Effects of liraglutide on weight loss, fat distribution, and $\beta$-Cell function in obese subjects with prediabetes or early type 2 diabetes," Diabetes Care, vol. 40, no. 11, pp. 1556-1564, 2017.

[3] G. Iacobellis and A. C. Villasante Fricke, "Effects of semaglutide versus dulaglutide on epicardial fat thickness in subjects with type 2 diabetes and obesity," Journal of the Endocrine Society, vol. 4, no. 4, article bvz042, 2020.

[4] A. M. Ansaldo, F. Montecucco, A. Sahebkar, F. Dallegri, and F. Carbone, "Epicardial adipose tissue and cardiovascular diseases," International Journal of Cardiology, vol. 278, no. 278, pp. 254-260, 2019.

[5] V. Miksztowicz, C. Morales, M. Barchuk et al., "Metalloproteinase 2 and 9 activity increase in epicardial adipose tissue of patients with coronary artery disease," Current Vascular Pharmacology, vol. 15, no. 2, pp. 135-143, 2017.

[6] F. Aboukhoudir, S. Rekik, M. Pansieri, and P. Obert, "Adipose epicardial tissue association with subclinical systolic dysfunction detected by longitudinal strain in diabetic patients with poor glycemic control," Annales de cardiologie et d'angeiologie, vol. 67 , no. 5, pp. 310-314, 2018.

[7] S. Morano, E. Romagnoli, T. Filardi et al., "Short-term effects of glucagon-like peptide 1 (GLP-1) receptor agonists on fat distribution in patients with type 2 diabetes mellitus: an ultrasonography study," Acta Diabetologica, vol. 52, no. 4, pp. 727 732, 2015.

[8] R. Bouchi, Y. Nakano, T. Fukuda et al., "Reduction of visceral fat by liraglutide is associated with ameliorations of hepatic steatosis, albuminuria, and micro-inflammation in type 2 diabetic patients with insulin treatment: a randomized control trial," Endocrine Journal, vol. 64, no. 3, pp. 269-281, 2017.

[9] W. A. Nuffer and J. M. Trujillo, "Liraglutide: a new option for the treatment of obesity," Pharmacotherapy, vol. 35 , no. 10 , pp. 926-934, 2015.

[10] J. Liu, G. Wang, Y. Jia, and Y. Xu, "GLP-1 receptor agonists: effects on the progression of non-alcoholic fatty liver disease," Diabetes/Metabolism Research and Reviews, vol. 31, no. 4, pp. 329-335, 2015.

[11] J. Seufert and B. Gallwitz, "The extra-pancreatic effects of GLP-1 receptor agonists: a focus on the cardiovascular, gastrointestinal and central nervous systems," Diabetes, Obesity of Metabolism, vol. 16, no. 8, pp. 673-688, 2014.

[12] G. Svegliati-Baroni, S. Saccomanno, C. Rychlicki et al., "Glucagon-like peptide-1 receptor activation stimulates hepatic lipid oxidation and restores hepatic signalling alteration induced by a high-fat diet in nonalcoholic steatohepatitis," Liver International, vol. 31, no. 9, pp. 1285-1297, 2011.

[13] M. Shah and A. Vella, "Effects of GLP-1 on appetite and weight," Reviews in endocrine metabolic disorders, vol. 15, no. 3, pp. 181-187, 2014.

[14] A. Garber, R. Henry, R. Ratner et al., "Liraglutide versus glimepiride monotherapy for type 2 diabetes (LEAD-3 Mono): a 
randomised, 52-week, phase III, double-blind, paralleltreatment trial," Lancet, vol. 373, no. 9662, pp. 473-481, 2009.

[15] K. Aoki, H. Kamiyama, M. Takihata et al., "Effect of liraglutide on lipids in patients with type 2 diabetes: a pilot study," Endocrine Journal, vol. 67, no. 9, pp. 957-962, 2020.

[16] S. P. Marso, G. H. Daniels, K. Brown-Frandsen et al., "Liraglutide and cardiovascular outcomes in type 2 diabetes," The New England Journal of Medicine, vol. 375, no. 4, pp. 311-322, 2016.

[17] H. S. Sacks, J. N. Fain, B. Holman et al., "Uncoupling protein-1 and related messenger ribonucleic acids in human epicardial and other adipose tissues: epicardial fat functioning as brown fat," The Journal of Clinical Endocrinology and Metabolism, vol. 94, no. 9, pp. 3611-3615, 2009.

[18] G. Iacobellis and G. Barbaro, "The double role of epicardial adipose tissue as pro- and anti-inflammatory organ," Hormone and Metabolic Research, vol. 40, no. 7, pp. 442-445, 2008.

[19] G. Iacobellis, D. Corradi, and A. M. Sharma, "Epicardial adipose tissue: anatomic, biomolecular and clinical relationships with the heart," Nature Clinical Practice. Cardiovascular Medicine, vol. 2, no. 10, pp. 536-543, 2005.

[20] C. Sengul and O. Ozveren, "Epicardial adipose tissue: a review of physiology, pathophysiology, and clinical applications," Anadolu Kardiyoloji Dergisi, vol. 13, no. 3, pp. 261-265, 2013.

[21] D. Bos, R. Shahzad, T. van Walsum et al., "Epicardial fat volume is related to atherosclerotic calcification in multiple vessel beds," European Heart Journal Cardiovascular Imaging, vol. 16, no. 11, pp. 1264-1269, 2015.

[22] G. HX-hT, "Relationship between visceral fat distribution and coronary damage in abdominal obesity adults," Journal of Chinese Practical Diagnosis and Therapy, vol. 1, 2018.

[23] S. A. Porter, J. M. Massaro, U. Hoffmann, R. S. Vasan, C. J. O'Donnel, and C. S. Fox, "Abdominal subcutaneous adipose tissue: a protective fat depot?," Diabetes Care, vol. 32, no. 6, pp. 1068-1075, 2009.

[24] S. N. Verhagen and F. L. Visseren, "Perivascular adipose tissue as a cause of atherosclerosis," Atherosclerosis, vol. 214, no. 1, pp. 3-10, 2011.

[25] Z. H. O. U. X. TC, Y. A. N. G. Guifen, and Z. H. U. Hong, "Progression of pericardial adipose tissue imaging," International Journal of Medical Radiology, vol. 43, no. 4, pp. 447-451, 2020.

[26] W. J. Zhang Hongmin, Z. Xingyu, and Y. Mei, "Effects of liraglutide combined with metformin on islet beta cell function and visceral fat level in type 2 diabetes mellitus patients with obesity," Chinese Journal of Cell and Stem Cell, vol. 9, no. 3, pp. 144-148, 2019.

[27] C. Sardu, N. D’Onofrio, M. Torella et al., "Pericoronary fat inflammation and Major Adverse Cardiac Events (MACE) in prediabetic patients with acute myocardial infarction: effects of metformin," Cardiovascular Diabetology, vol. 18, no. 1, p. 126, 2019.

[28] R. Marfella, C. Amarelli, F. Cacciatore et al., "Lipid accumulation in hearts transplanted from nondiabetic donors to diabetic recipients," Journal of the American College of Cardiology, vol. 75, no. 11, pp. 1249-1262, 2020.

[29] G. Iacobellis, M. Mohseni, S. Bianco, and P. Banga, "Liraglutide causes large and rapid epicardial fat reduction," Obesity, vol. 25, no. 2, pp. 311-316, 2017.
[30] J. Jonker, H. Lamb, R. van der Meer et al., "Pioglitazone compared with metformin increases pericardial fat volume in patients with type 2 diabetes mellitus," The Journal of Clinical Endocrinology and Metabolism, vol. 95, no. 1, pp. 456-460, 2010.

[31] M. Ziyrek, S. Kahraman, E. Ozdemir, and A. Dogan, “A monoterapia com metformina diminiu significativamente a espessura do tecido adiposo epicardico nos doentes recentemente diagnosticados com diabetes tipo 2," Revista Portuguesa de Cardiologia, vol. 38, no. 6, pp. 419-423, 2019.

[32] M. Bizino, I. Jazet, P. de Heer et al., "Placebo-controlled randomised trial with liraglutide on magnetic resonance endpoints in individuals with type 2 diabetes: a pre-specified secondary study on ectopic fat accumulation," Diabetologia, vol. 63, no. 1, pp. 65-74, 2020.

[33] E. Dozio, E. Vianello, A. E. Malavazos et al., "Epicardial adipose tissue GLP-1 receptor is associated with genes involved in fatty acid oxidation and white-to-brown fat differentiation: a target to modulate cardiovascular risk?," International journal of cardiology, vol. 292, pp. 218-224, 2019.

[34] S. Steven, K. Jurk, M. Kopp et al., "Glucagon-like peptide-1 receptor signalling reduces microvascular thrombosis, nitrooxidative stress and platelet activation in endotoxaemic mice," British Journal of Pharmacology, vol. 174, no. 12, pp. 16201632, 2017.

[35] J. Helmstädter, K. Frenis, K. Filippou et al., "Endothelial GLP1 (glucagon-like peptide-1) receptor mediates cardiovascular protection by liraglutide in mice with experimental arterial hypertension," Arteriosclerosis, Thrombosis, and Vascular Biology, vol. 40, no. 1, pp. 145-158, 2020.

[36] J. Helmstädter, K. Keppeler, F. Aust et al., "GLP-1 analog liraglutide improves vascular function in polymicrobial sepsis by reduction of oxidative stress and inflammation," Antioxidants, vol. 10, no. 8, p. 1175, 2021.

[37] G. Mansueto, D. Costa, E. Capasso et al., "The dating of thrombus organization in cases of pulmonary embolism: an autopsy study," BMC Cardiovascular Disorders, vol. 19, no. 1, p. 250, 2019. 